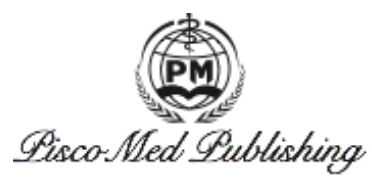

Original Research Article

\title{
Machine Learning Applied in the Financial Industry
}

\author{
Yuntao Sun* \\ University of California, Irvine, Irvine, CA92697, United States. E-mail: yuntaos1@uci.edu
}

\begin{abstract}
Technological development provides industries and spheres with numerous benefits, particularly availability of new progressive methods that contribute to increase efficiency and enhance performance. Thus, machine learning methods may contribute to financial industry that is involved in processing of a large volume of data. Machine learning methods facilitate to process data faster and efficiently with the minimal intervention of humans. In addition, it helps to predict possible risks for financial business and minimize risks related to the fraudulent activity or financial losses. Furthermore, application of machine learning methods contributes to enhance the work with clients and targeted groups, as well as provide them with appropriate services. The major risks of machine learning methods applications within the financial sphere relate to unpredictability and cyber security issues.
\end{abstract}

Keywords: Method; Financial Industry; Machine Learning; Data

\section{New method applied in the financial industry}

The financial industry is a data-driven business that requires reliable and high-quality data. In fact, the quality and reliability of data depend directly on the methods that analysts and experts apply to collect, organize, and analyze data. Currently, businesses and financial institutions have numerous challenges related to the large volume of data processing, which is collected most often from databases, the Internet recourses, or financial apps. Moreover, how to analyze these data efficiently can also become a problem. Therefore, a method named machine learning has become increasingly popular within the financial sphere to tackle issues involving large amounts of data. Application of machine learning methods in the financial sphere facilitate the financial industry to make better decisions, operate efficiently with a large volume of data, improve business processes, apply business data more efficiently and timely regardless of possible risks that relate to the application of the machine learning methods in the financial industry.

\section{Notion, application and importance of machine learning methods}

Most of the data that companies collected from the website are clickstream data, which are building up with many other small data branches including visits, time of site, page views, and bounce rate. However, these data only tell companies 'what', but they didn't tell the companies 'why'? And focusing on one kind of data cannot provide sufficient information to help companies to make wise decisions, companies need to combine these data and analysis them together in order to dig the really valid and helpful information. Moreover, the problem here is the whole process is easier said than done since each data branch contains too much numerous and trivial data that are hard to be efficiently processed by traditional methods. But Machine learning methods provide companies automotive analytical model building to analyze data which can be a huge advantage while making business decisions. According to Jordan and Mitchell

Copyright (C) 2020 Yuntao Sun

doi: $10.18282 /$ ff.v9i4.1554

This is an open-access article distributed under the terms of the Creative Commons Attribution Non-Commercial License

(http://creativecommons.org/licenses/by-nc/4.0/), which permits unrestricted non-commercial use, distribution, and reproduction in any medium,

provided the original work is properly cited. 
$(2015)^{[1]}$, machine learning is based on the concept that systems can learn from data, identify patterns, and make efficient decisions with minimal people intervention. In addition, Murdoch et al. (2019) ${ }^{[2]}$ state that machine learning methods provide the ability to use innovative technologies and improve the operation and processing of data and important business information. According to the consideration of Dixon, Haplerin, and Bilokon (2020 $)^{[3]}$, machine learning methods stipulate machines to learn, make predictions, and improve the algorithms on their own that contributes to the business performance and increased efficiency.

The most popular machine learning methods in the financial sphere are supervised and unsupervised learning. Supervised learning stipulates usage of labeled algorithms and includes Linear Regression, LASSO, RIDGE, Polynomial Regression, CHAID, Random Forest, KNN, Neural Network, C\&RT (Xie, 2019) ${ }^{[4]}$. For instance, data could have labels either "F" (false) or "R" (right). The machine learning algorithm has a set of inputs and outputs (Xie, 2019) ${ }^{[4]}$. In fact, the algorithm works on the principle to compare an actual output with the correct one (Xie, 2019) ${ }^{[4]}$. Further, it modifies the model accordingly by means of classification, prediction, or regression (Xie, 2019) ${ }^{[4]}$. Supervised learning methods use samples to predict the values (Xie, 2019) ${ }^{[4]}$. For instance, supervised learning methods can predict which customer is likely to conduct fraudulent actions or file a claim. The unsupervised learning method is based on the opposite algorithm that figures out what is being shown (Xie, 2019) ${ }^{[4]}$. The most popular methods include K-means, Hierarchical Classification, Two Step, K-modes, and X-means (Xie, 2019) ${ }^{[4]}$. According to Xie (2019) ${ }^{[4]}$, the objective of the unsupervised learning method is to explore the data and find a similar structure. For example, it can show a group of clients that have similar attributes or can show distinctive features that separate clients.

The practice of using machine learning methods by financial institutions in their activities is widespread worldwide. Banks and other business entities use machine learning methods for two key reasons: to prevent fraud and identify important information in data. For instance, MasterCard uses the machine learning method to track and process transaction size, device, location, time, and purchase data. In addition, machine learning methods contribute to identify investment opportunities, or provide important and valuable information for investors. Data mining by means of machine learning methods can identify clients with high-risk profiles, or provide information appropriate to predict or mitigate risks of fraud. In addition, machine learning methods facilitate granting credit (Murdoch et al., 2019) ${ }^{[2]}$. Thus, Zest Finance facilitates organizations to assess borrowers with little credit history. Underwrite.ai provides an analysis of data that is received from credit bureau sources with the aim to evaluates credit risk for clients or possible risks for small business loan applicants. Data Robot helps to organize and systemize data related to fraudulent credit card transactions, direct marketing, or lending.

The application of machine learning methods provides the industry of finance with benefits. In most business environments, machine learning methods are being applied to get advantages of time consumption, cutting off cost, and furthermore bringing in added values. Machine learning methods contribute to the efficient processing of growing volumes of available data (Hamdoun \& Rguibi, 2019) ${ }^{[5]}$. Furthermore, data processing by means of machine learning methods is much cheaper and efficient (Hamdoun \& Rguibi, 2019) ${ }^{[5]}$. According to Hamdoun and Rguibi (2019) ${ }^{[5]}$, machine learning methods make it possible to establish models that can analyze bigger and more complex data, as well as deliver more accurate results faster and automatically. In addition, business entities that apply machine learning methods have high chances to identify profitable opportunities and avoid possible risks. Thus, machine learning methods are of great importance for the financial industry, particularly for their advantages.

\section{Advantages of machine learning methods}

Financial organizations are thinking about the application of machine learning methods since it has associated with a variety of advantages (see figure 1). Organizations can make efficient decisions without human intervention by using machine learning methods (Jordan and Mitchell, 2015) ${ }^{[1]}$. In addition, machine learning algorithms are beneficial to process multi-dimensional data in the dynamic environment (Murdoch et al., 2019) ${ }^{[2]}$. The methods facilitate to review and adjust messages based on recent customer interactions and behaviors (Murdoch et al., 2019) ${ }^{[2]}$. Once a model is forged from multiple data sources, it has the ability to pinpoint relevant variables (Murdoch et al., 2019) ${ }^{[2]}$. Thus, it 
prevents from complicated integrations, while focusing only on concise and precise data.

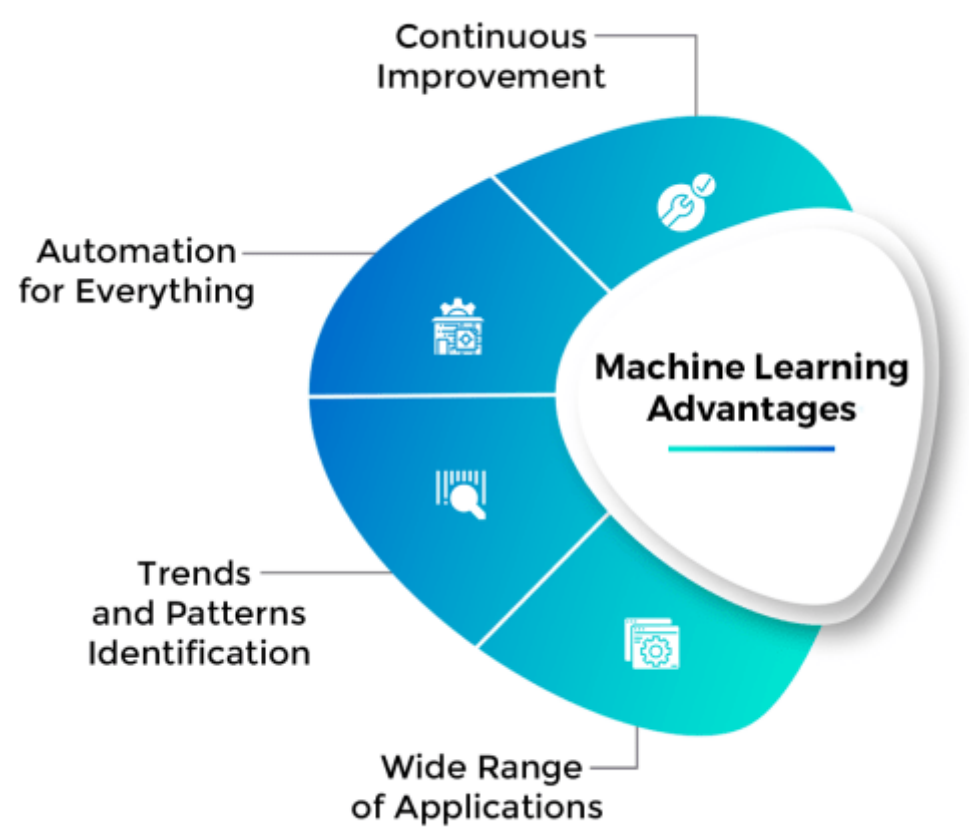

Figure 1. Advantages of machine learning methods application

The application of machine learning methods is important in the financial sphere. While predictive analytics is instrumental in saving costs and building revenue - it is equally as important to understand their impacts on real-life situations pertaining to clients' acquisitions or loss (Hamdoun \& Rguibi, 2019) ${ }^{[5] .}$ In fact, systems are able to quickly act upon the outputs of machine learning - making marketing message and data processing more effective (Hamdoun \& Rguibi, 2019) ${ }^{[5]}$. For example, newly obtained data may contribute businesses to present new offers for targeted clients. However, data can also signify cutting back on unnecessary offers if these customers do not require them for conversion purposes.

On the other hand, there are certain risks that relate to the application of machine learning methods in the financial sphere.

\section{Risks of application}

The major risks of machine learning methods application are unpredictability, cybersecurity issues, risk of players' dependency, and risk of financial instability (see figure 2). According to Jadhav and Jenkins (2016) ${ }^{[6]}$, machine learning methods may be less predictable than current rule-based applications and may interact in unexpected ways, may also enhance the interconnectedness of financial markets and institutions in unexpected ways. In addition, machine learning increases possible attack points and new attacks are designed to alter the functioning of business algorithms (Jordan \& Mitchell, 2015) ${ }^{[1]}$. Another challenge is the ability to interpret results generated by the algorithms accurately (Jordan \& Mitchell, 2015) ${ }^{[1]}$. Organizations should carefully choose the algorithms for certain purposes. 


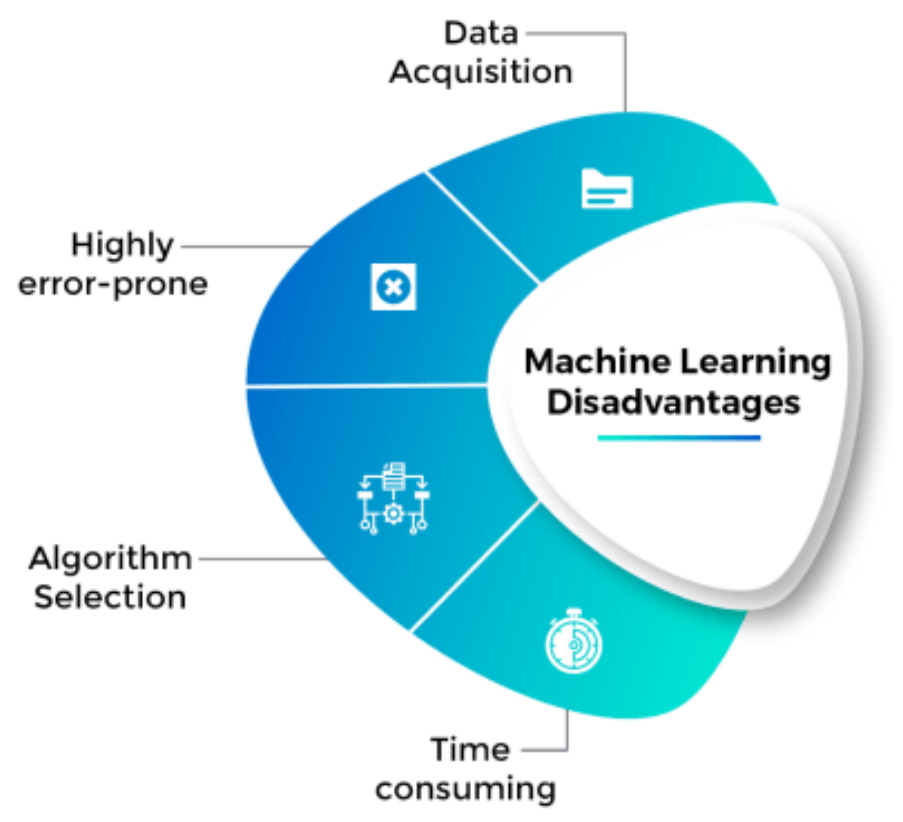

Figure 2. Disadvantages of machine learning methods application

In addition, according to Xie (2019) $)^{[4]}$, machine learning is highly susceptible to errors, as well as requires specific resources application. In the process of machine learning, a high amount of data is used, as well as many algorithms are used (Xie, 2019) ${ }^{[4]}$. However, the business should clearly state objectives and apply appropriate algorithms with an aim to mitigate the emergence of failures. Furthermore, machine learning requires time to let the algorithms learn and fulfill business' objectives with accuracy and relevancy (Xie, 2019) ${ }^{[4]}$. It also needs specific resources to function, particularly innovative technology application (Xie, 2019) ${ }^{[4]}$. Thus, application of machine learning methods requires businesses to evaluate all possible risks with the aim to get results.

\section{Conclusion}

Financial data are being generated rapidly. Therefore, business requires reliable methods appropriate to process data efficiently. A lot of investment decisions in the area of the finance industry are supported by machine learning methods that contribute to the finance industry, particularly to collect, analyze the data, as well as gain a competitive advantage from the data. Machine learning methods provide the ability to use innovative technologies and improve the operation and processing of the data and important business information. The application of machine learning method provides the industry of finance with benefits. In most of the business aspects, machine learning methods are being applied to acquire the advantages of time consumption, cutting off costs, and bringing in added values. The most often used methods in the financial industry are Linear Regression, LASSO, RIDGE, Polynomial Regression, Two Step, K-modes. The major risks of machine learning methods application are unpredictability, cybersecurity issues, risk of players' dependency, and risk of financial instability.

\section{References}

1. Jordan MI, Mitchell TM. Machine learning: Trends, perspectives, and prospects. Science 2015; 349(6245): 255- 
260.

2. Murdoch WJ, Singh C, Kumbier K, et al. Definitions, methods, and applications in interpretable machine learning. Proceedings of the National Academy of Sciences 2019; 116(44): 22071-22080.

3. Dixon MF, Halperin I, Bilokon P. Machine learning in finance: From theory to practice. Switzerland: Springer Nature Switzerland AG; 2020.

4. Xie M. Development of artificial intelligence and effects on financial system. Journal of Physics: Conference Series 2019; 1187(3): 032084.

5. Hamdoun N, Rguibi K. Impact of AI and machine learning on financial industry: application on Moroccan credit risk scoring. Journal of Advanced Research in Dynamical and Control Systems 2019; 11(11): 1041-1048.

6. Jadhav S, He H, Jenkins K. An academic review: Applications of data mining techniques in finance industry. International Journal of Soft Computing and Artificial Intelligence, 2016; 4(1): 79-95. 